\title{
Template Composite Dark Matter : $S U(2)$ gauge theory with 2 fundamental flavours.
}

\author{
V. Drach ${ }^{* a, b}$, A. Hietanen ${ }^{a}$, C. Pica ${ }^{a}$, J. Rantaharju ${ }^{a}$, F. Sannino ${ }^{a}$ \\ ${ }^{a} C^{3}$-Origins \& the Danish Institute for Advanced Study DIAS, University of Southern Denmark, \\ Campusvej 55, DK-5230 Odense M, Denmark \\ ${ }^{b}$ CERN, Physics Department, 1211 Geneva 23, Switzerland \\ E-mail:vincent.drach@cern.ch
}

\begin{abstract}
We present a non perturbative study of SU(2) gauge theory with two fundamental Dirac flavours. We discuss how the model can be used as a template for composite Dark Matter (DM). We estimate one particular interaction of the DM candidate with the Standard Model : the interaction through photon exchange computing the electric polarizability of the DM candidate. Finally, we briefly discuss the viability of the model given the present experimental constraints.
\end{abstract}

Preprint : CP3-Origins-2015-046 DNRF90 \& DIAS-2015-46

The 33rd International Symposium on Lattice Field Theory

14 -18 July 2015

Kobe International Conference Center, Kobe, Japan*

\footnotetext{
* Speaker.
} 


\section{Introduction}

The fact that $25 \%$ of the energy content of the Universe must be accounted for by Dark Matter (DM) is confirmed by several experiments. Many models have been built to describe the Dark Matter sector of our Universe and are severely constraints by experimental data obtained directly, indirectly, or using colliders. One interesting scenario is the one of Composite Dark Matter where, as in baryonic sector, the most abundant particles are "composite". In this work we consider one particular realization of a composite dark matter model that breaks electroweak symmetry dynamically[1]. The Dark matter candidate is electrically neutral and its interactions with nucleon receive contributions from Higgs-exchange, from the electric dipole moment (which vanishes in the limit of degenerate fermions), and at higher order in the operator expansion from a two-photon exchange vertex, as illustrated in Fig. 1. While the two first contributions have already been investigated in [2], we compute here the latter contribution and estimate the cross section relevant for direct detection experiments. Note that such an interaction has also be considered on the lattice in the context of Stealth Dark Matter[4].

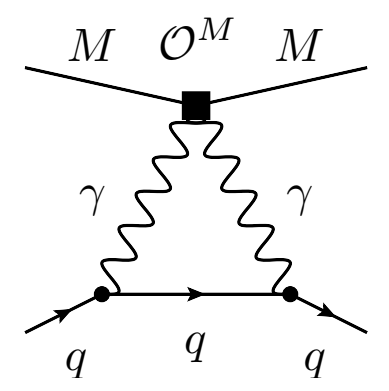

Figure 1: Illustration of a two-photon interaction with quarks[3].

The model is based on $S U(2)$ gauge field theory with two fermions in the fundamental representation. The Lagrangian reads in the continuum :

$$
\mathscr{L}=-\frac{1}{4} F_{\mu v}^{a} F^{a \mu v}+\bar{\psi}(i \not D-m) \psi,
$$

where $\psi=(u, d)$ is a doublet of Dirac spinor fields transforming according to the fundamental representation and can also be written as :

$$
\left.\mathscr{L}=-\frac{1}{4} F_{\mu v}^{a} F^{a \mu v}+\bar{\psi} i \not D \psi+\frac{i m}{2}\left[Q^{T}\left(-i \sigma_{2}\right) C E Q+\left(Q^{T}\left(-i \sigma_{2}\right) C E Q\right)\right)^{\dagger}\right]
$$

where $\sigma_{2}$ acts on color indices and $C$ is the charge conjugation matrix. Furthermore, we have defined :

$$
Q=\left(\begin{array}{c}
u_{L} \\
d_{L} \\
-i \sigma_{2} C \bar{u}_{R}^{T} \\
-i \sigma_{2} C \bar{d}_{R}^{T}
\end{array}\right), \text { and } E=\left(\begin{array}{cccc}
0 & 0 & +1 & 0 \\
0 & 0 & 0 & +1 \\
-1 & 0 & 0 & 0 \\
0 & -1 & 0 & 0
\end{array}\right)
$$

We have used $q_{L, R}=P_{L, R} q, \bar{q}_{L, R}=\bar{q} P_{R, L}$ with $P_{L}=\frac{1}{2}\left(1-\gamma_{5}\right)$ and $P_{R}=\frac{1}{2}\left(1+\gamma_{5}\right)$. The model exhibits an $S U$ (4) flavour symmetry in the massless limit. The 15 generators of the corresponding 
Lie algebra will be denoted $T^{a=1, \ldots, 15}$. After adding a mass term, the remnant flavour symmetry is the group spanned by the algebra that preserves $E T^{a, T}+T^{a, T} E=0$. This relation defines the 10dimensional algebra of the $S P(4)$ group. The chiral symmetry breaking pattern is thus expected to be $S U(4) \rightarrow S P(4)$ leading to 5 Goldstone bosons. The model has been investigated on the lattice in [5], and the chiral symmetry breaking pattern has been proven to be the expected one [6].

As proposed in [1], the Lagrangian Eq. (1.1) can be embedded into the Standard Model in such a way that it interpolates between composite Goldstone Higgs and Technicolor models[7, 8]. The two limits are parametrized by a single parameter whose value depends on contribution from Standard Model loops. In the so-called technicolor limit the model breaks electroweak symmetry and 3 of the Goldstone bosons provide mass to W's and Z gauge bosons while the two remaining Goldstone bosons are stable and can be arranged in a electrically neutral complex scalar field, denoted $\phi$, which is a Dark Matter candidate. As argued in [1], the Dark Matter mass is generated via loop diagrams involving electroweak bosons and top quarks, they predict that the mass is proportional to the scale $f_{\Pi}=246 \mathrm{GeV}$, and we will thus restrict ourselves to $m_{\phi}<500 \mathrm{GeV}$. Note that the $S U(2)$ gauge theory with two fundamental fermions have been used to build several Dark Matter models[4, 9, 10, 11, 12].

In this model the two quark $\left(U_{L}, D_{L}\right)$ are arranged in a $S U(2)_{L}$ doublet with hypercharge 0 , and the two remaining (Weyl) fermions are singlet of $S U(2)_{L}$ with hypercharge $\pm 1 / 2$. The electric charge matrix of the fermions is $\mathscr{Q}=\operatorname{diag}\left(\frac{1}{2},-\frac{1}{2},-\frac{1}{2}, \frac{1}{2}\right)$.

\section{Electromagnetic properties}

Since the underlying fermions are not electrically neutral, the effective theory describing the composite (Goldstone) Dark Matter candidate is expected to generate a two-photon coupling. The goal of this work is to investigate that particular contribution. The low energy coupling constant that enter into the process $\phi \phi \rightarrow \gamma \gamma$ is called the polarizabity (measured in $[\mathrm{fm}]^{3}$ ) and enter in the compton cross section which can be computed in chiral perturbation theory. In order to perform a lattice calculation, a different approach is followed. As shown in [13], the polarizability also characterizes the response of the mass of a spin- 0 neutral bound state to a classical constant electromagnetic field according to the following small field expansion :

$$
m^{(\mathscr{E})}=m^{0}+\frac{1}{2} 4 \pi \alpha_{E} \mathscr{E}^{2}+\ldots,
$$

where $\alpha_{E}$ is the electromagnetic polarizability. The latter relation suggests a first principle approach to calculate the polarizability and has been used in QCD to determine polarizabilities of various hadrons, see for instance [14]. We briefly sketch the method in the following. In order to fulfill the 't Hooft condition[15], the electric field needs to be quantized according to

$$
\mathscr{E}=\left(e a^{2}\right)^{-1} \frac{2 \pi n}{Q N_{t} N_{L}} \equiv\left(e a^{2}\right)^{-1} E,
$$

where $e$ is related to the electromagnetic coupling constant $\alpha=e^{2} /(4 \pi), a$ is the lattice spacing, $Q$ the charge of fermion, $n$ is an integer, and $N_{t}, N_{l}$ are the temporal and spatial lattice extent. Note furthermore that we have introduced the dimensionless lattice field $E$. The gauge links are then multiplied by a position dependent field $U_{\mu}^{(E)}$ defined as follows :

$$
U_{\mu}^{(E)}=e^{i \mathscr{2} A_{\mu}(x)} e^{i Q E N_{t} x_{3} \delta_{\mu, 4} \delta_{x_{4}, N_{t}-1}}, \quad \text { where } \quad A_{\mu}(x)=\left(0,0,-E x_{4}, 0\right),
$$


$\mathscr{Q}$ is the electric charge of the fermion, $N_{t}$ is the lattice temporal extent. In our calculation, the electromagnetic background field is included only in the valence which leads to a systematic uncertainty. We however expect the calculation to provide an order of magnitude estimate of the polarizability.

In our model the dark matter candidate has the same quantum numbers as the following diquark operator $u^{T} C \gamma_{5} \sigma_{2} d$. The mass as function of the electric field can be estimated by computing the effective mass corresponding to the following two-point function :

$$
C_{2 \mathrm{pt}}^{\mathscr{E}}(t)=\sum_{\vec{x}}\left\langle\phi(x) \phi^{\dagger}(0)\right\rangle=C_{2 \mathrm{pt}, \mathrm{conn} .}^{\mathscr{E}, \pi^{0}}(t) .
$$

The last equality -which relates $C_{2 \mathrm{ft}}^{\mathscr{E}}(t)$ to the connected part of the neutral pion two-point functioncan be derived using properties of the Wilson-Dirac operator for a two-color theory in a presence of a background field.

Note that by inspecting the corresponding effective theory, we concluded that a polarizability operator is generated at order $\mathscr{O}\left(E^{6}\right)$ by an operator of the form $m_{\phi}^{2} F_{\mu v} F^{\mu v} \phi^{*} \phi$. The polarizability is thus expected to vanish in the chiral limit. Note that $F_{\mu \nu} F^{\mu v} \partial_{\rho} \phi \partial^{\rho} \phi$ also appears in the effective theory. The latter is not expected to vanish in the chiral limit and will thus dominate the cross section for very light DM mass. We will disregard that contribution in this preliminary study.

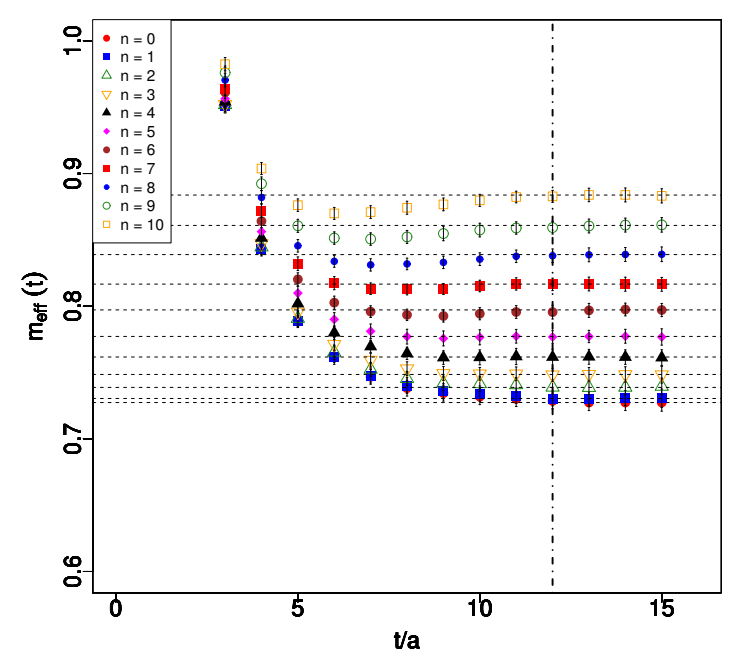

Figure 2: Effective masses for $\beta=2.2, L=16^{3} \times$ 32 and $m_{0}=-0.65$ for values of the electric field corresponding to $n=0, \ldots, 10$.

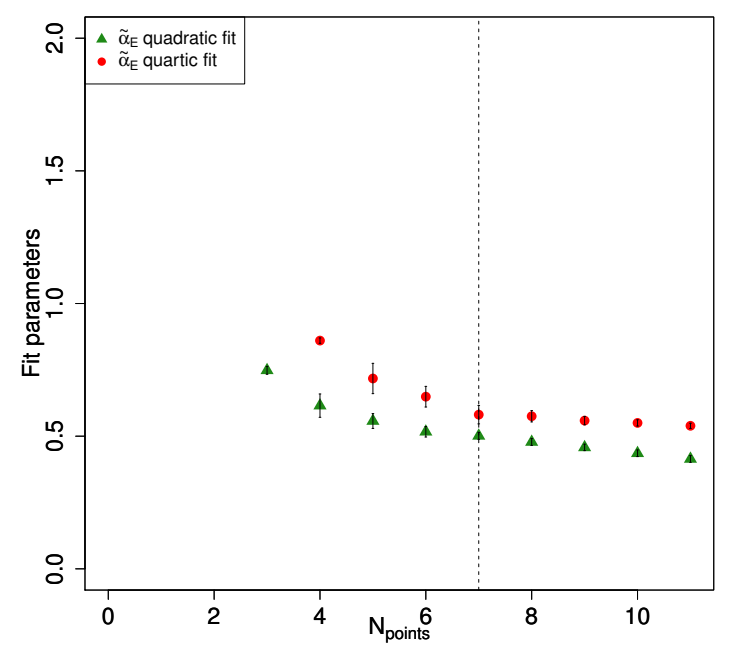

Figure 3: $\widetilde{\alpha}_{E}$ assuming a quadratic or a quartic fit ansätz as a function of the number of points included in the fit for $\beta=2.2, L=16^{3} \times 32$ and $m_{0}=-0.65$

\section{Results}

The simulation are performed using two flavour Wilson fermions with the plaquette action. Two lattice spacing are used in this work and the pseudoscalar decay constant $f_{\mathrm{PS}}$ is renormalized perturbatively. Furthermore since the we are considering only the case $\theta=\pi / 2$ (technicolor limit), the scale is set by imposing $F_{\Pi}=246 \mathrm{GeV}$. 
In Fig. 2 we show the effective mass defined by solving the equation

$$
\frac{C(t-a)}{C(t)}=\frac{e^{-m_{\mathrm{eff}}(t)(t-a)}+e^{-m_{\mathrm{eff}}(t)(T-(t-a))}}{e^{-m_{\mathrm{eff}}(t) t}+e^{-m_{\mathrm{eff}}(t)(T-t)}},
$$

for values of the electric field with $n=0, \ldots, 10$. The results show clear plateaus and the masses are obtained by fitting the effective masses for $t / a$ larger than the vertical dotted line. Once the mass has been determined for each value of $n$, the dimensionless electric field dependence is fitted according to :

$$
a M^{(E)}=a M^{0}+\frac{1}{2} 4 \pi \widetilde{\alpha}_{E} E^{2}+\mathscr{O}\left(E^{4}\right), \quad \text { where } \quad \widetilde{\alpha}_{\mathrm{E}}=\frac{\alpha_{\mathrm{E}}}{4 \pi \alpha \mathrm{a}^{3}} .
$$

The relation between $\widetilde{\alpha}_{E}$ and $\alpha_{E}$ can be derived by matching Eq. (2.1) and using the definition Eq. (2.2). We show in Fig. 3 the fitted value of $\widetilde{\alpha}_{E}$ assuming a quadratic or a quartic fit ansätz as a function of the number of points included in the fit $\left(N_{\text {points }}\right)$. For $N_{\text {points }}$, the two estimations of $\widetilde{\alpha}_{E}$ are of the same order of magnitude indicating that the contribution from the quartic coefficient is small. Also, the value of $\widetilde{\alpha}_{E}$ does not depend strongly on $N_{\text {points }}$ and the fit is thus stable. Note furthermore that the expansion variable of Eq. (2.1): $\frac{(e \mathscr{E})^{2}}{m_{\mathrm{PS}}^{4}} \sim 0.03 n^{2}$ is small even for the smallest quark mass used in our setup.

In Fig. 4, we show the dependence of $\widetilde{\alpha}_{E}$ as a function of $m_{\mathrm{PS}} L$ for two ensembles at fixed $\beta=2.2$. Note that the lightest fermion mass is included in the plot $\left(m_{0}=-0.75\right)$. Since our results do not depend significantly of the volume, we conclude that finite volume effects are negligible in our results. In Fig. 5, we show the dimensionless quantity $\alpha_{E} m_{\mathrm{PS}} f_{\mathrm{PS}}^{2}$ as a function of $m_{\mathrm{PS}}^{2} \equiv m_{\phi}^{2}$ at two different lattice spacing. We choose that particular combination because we expect it to cancel the leading order behaviour of $\alpha_{E}$. Note that the results obtained at $\beta=2.0$ show that our results are not significantly affected by lattice $\mathscr{O}(a)$ effects. They are thus safely neglected in the following. We performed a polynomial fit of $\alpha_{E} m_{\mathrm{PS}} f_{\mathrm{PS}}^{2}$ imposing that $\alpha_{E} m_{\mathrm{PS}} f_{\mathrm{PS}}^{2}$ vanishes in the chiral limit as required by the effective field theory. The best fit value obtained at fixed $\beta=2.2$ is depicted by a dotted line. Using the relation between $f_{\mathrm{PS}}$ and $m_{\mathrm{PS}}$ from our previous work [5], we deduce a prediction for $\alpha_{E}\left(m_{\phi}^{2}\right)$.

Following [4], the effective interaction Lagragian between DM and photons can be written $\mathscr{L}=\pi \alpha_{E} F_{\mu v} F^{\mu v} \phi^{*} \phi$ and the cross section per nucleon for a given target with atomic and mass number $(Z, A)$ can be written as follows :

$$
\sigma_{\text {nucleon }}(Z, A)=\frac{Z^{4}}{A^{2}} \frac{9 \pi \alpha^{2} \mu_{n \phi}^{2}\left(M_{F}^{A}\right)^{2}}{R^{2}} \alpha_{E}^{2},
$$

where $\mu_{n \phi}$ is the reduced mass, $\alpha$ is the electromagnetic coupling constant, $R=1.2 A^{1 / 3}$ and $1<M_{A}^{F}<3$ which enters in the nuclear part of the cross section. We refer to [4], for a detailed discussion of the assumption made to estimate the cross section.

Using our prediction of $\alpha_{E}\left(m_{\phi}^{2}\right)$ we plot by a blue band, whose width is determined by the large uncertainty on the matrix element $M_{A}^{F}$, the cross section per nucleon for Xenon in Fig. 6. In the figure, we also represented by a grey area the latest constraints obtained by the LUX experiments[16]. The orange filled area is the region of cross section were experiments are not able to discriminate a nucleon-DM event from a coherent neutrino recoil [17]. We conclude that, within our assumption, the cross section due to two photon exchange is orders of magnitude too small to be detected. 


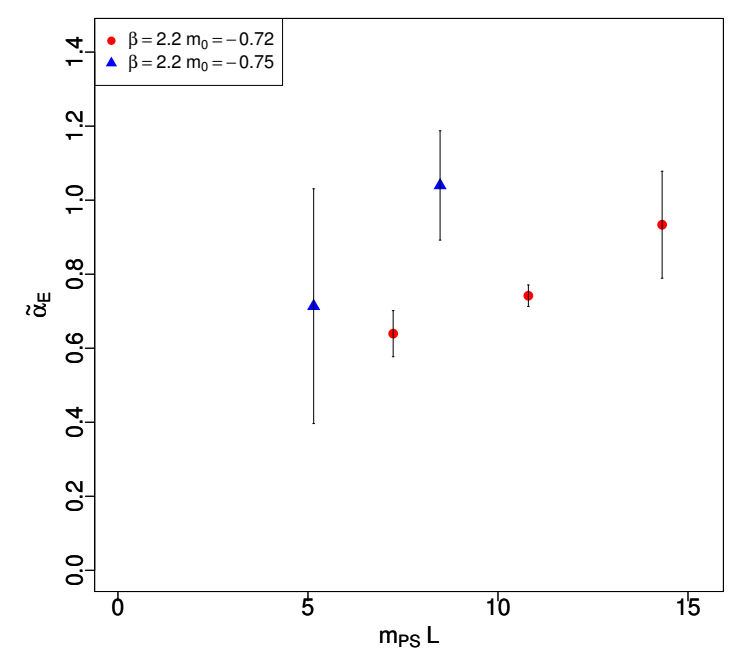

Figure 4: $\widetilde{\alpha}_{E}$ as a function of $m_{\mathrm{PS}} L$ for two ensembles at $\beta=2.2$, including the lightest fermion mass used in our simulation.

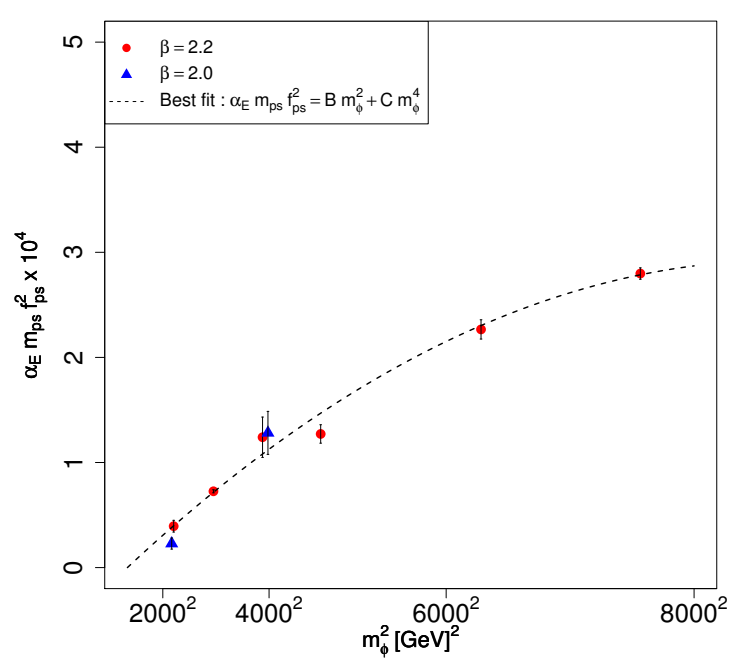

Figure 5: $\alpha_{E} m_{\mathrm{PS}} f_{\mathrm{PS}}^{2} \times 10^{4}$ as a function of $m_{\mathrm{PS}}^{2} \equiv$ $m_{\phi}^{2}$ at two different lattice spacing. A polynomial extrapolation including only $\beta=2.2$ results is depicted by a dotted line.

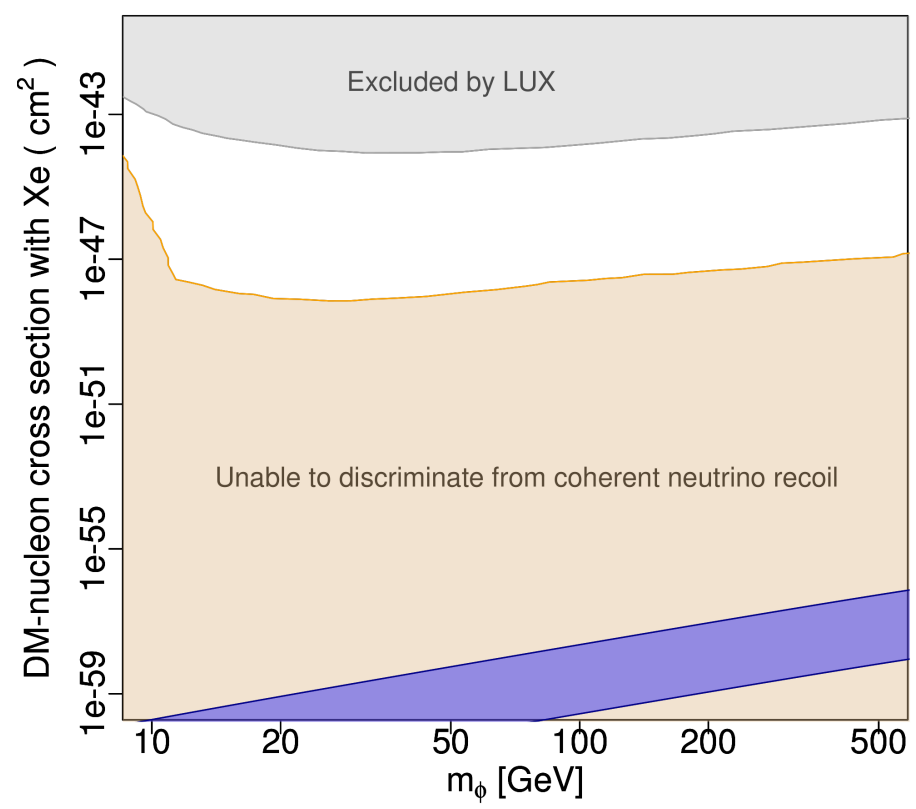

Figure 6: Prediction of the nucleon-DM cross section per nucleon for Xenon (blue band), constraints set by the LUX experiments[16], and region were experiments are not able to discriminate from coherent neutrino recoil[17]. 


\section{Conclusion}

We considered the interesting framework of a unified composite Higgs and technicolor models. We argued that in the technicolor limit the model features a DM candidate. Because of its composite nature, the DM candidate is sensitive to electromagnetic interaction via the electric polarizability of the DM. We performed a lattice calculation in isolation of the SM of the electric polarizability using the background field method. We concluded that the expected cross section is to small to be accessible via direct detection. In future works we plan to investigate what happens beyond the technicolor limit of the model and to study the effect of electroweak corrections to the nucleon-DM cross section.

\section{Acknowledgments}

This work was supported by the Danish National Research Foundation DNRF:90 grant and by a Lundbeck Foundation Fellowship grant. The computing facilities were provided by the Danish Centre for Scientific Computing.

\section{References}

[1] G. Cacciapaglia and F. Sannino, JHEP 1404, 111 (2014) [arXiv:1402.0233 [hep-ph]].

[2] A. Hietanen, R. Lewis, C. Pica and F. Sannino, JHEP 1412, 130 (2014) [arXiv:1308.4130 [hep-ph]].

[3] M. T. Frandsen, U. Haisch, F. Kahlhoefer, P. Mertsch and K. Schmidt-Hoberg, JCAP 1210, 033 (2012) [arXiv:1207.3971 [hep-ph]].

[4] T. Appelquist et al., arXiv:1503.04205 [hep-ph].

[5] A. Hietanen, R. Lewis, C. Pica and F. Sannino, JHEP 1407, 116 (2014) [arXiv:1404.2794 [hep-lat]].

[6] R. Lewis, C. Pica and F. Sannino, Phys. Rev. D 85, 014504 (2012) [arXiv:1 109.3513 [hep-ph]].

[7] T. A. Ryttov and F. Sannino, Phys. Rev. D 78, 115010 (2008) [arXiv:0809.0713 [hep-ph]].

[8] T. Appelquist, P. S. Rodrigues da Silva and F. Sannino, Phys. Rev. D 60, 116007 (1999) [hep-ph/9906555].

[9] W. Detmold, M. McCullough and A. Pochinsky, Phys. Rev. D 90, no. 11, 115013 (2014) [arXiv:1406.2276 [hep-ph]].

[10] W. Detmold, M. McCullough and A. Pochinsky, Phys. Rev. D 90, no. 11, 114506 (2014) [arXiv:1406.4116 [hep-lat]].

[11] Y. Hochberg, E. Kuflik, H. Murayama, T. Volansky and J. G. Wacker, Phys. Rev. Lett. 115, no. 2, 021301 (2015) [arXiv:1411.3727 [hep-ph]].

[12] M. Hansen, K. Langæble and F. Sannino, Phys. Rev. D 92, no. 7, 075036 (2015) [arXiv:1507.01590 [hep-ph]].

[13] B. C. Tiburzi, Nucl. Phys. A 814, 74 (2008) [arXiv:0808.3965 [hep-ph]].

[14] W. Detmold, B. C. Tiburzi and A. Walker-Loud, Phys. Rev. D 81, 054502 (2010) [arXiv:1001.1131 [hep-lat]].

[15] G. 't Hooft, Nucl. Phys. B 153, 141 (1979).

[16] D. S. Akerib et al. [LUX Collaboration], Phys. Rev. Lett. 112, 091303 (2014) [arXiv:1310.8214 [astro-ph.CO]].

[17] J. Billard, L. Strigari and E. Figueroa-Feliciano, Phys. Rev. D 89, no. 2, 023524 (2014) [arXiv:1307.5458 [hep-ph]]. 